\title{
Numerical crack growth study on porosity afflicted cast steel specimens
}

\author{
Manuel Schuscha, Martin Leitner, Michael Stoschka, Stefan Pusterhofer \\ Christian Doppler Laboratory for Manufacturing Process based Component Design, Montanuniversität Leoben, Chair of \\ Mechanical Engineering, Leoben, Austria \\ manuel.schuscha@unileoben.ac.at, bttp://orcid.org/0000-0001-9481-5275 \\ martin.leitner@unileoben.ac.at, bttp:/ / orcid.org/0000-0002-3530-1183 \\ michael.stoschka@unileoben.ac.at,bttp://orcid.org/0000-0003-4817-4432 \\ stefan.pusterbofer@unileoben.ac.at
}

\author{
Giovanni Meneghetti \\ University of Padova, Department of Industrial Engineering, Padova, Italy \\ giovanni.meneghetti@unipd.it, bttp://orcid.org/0000-0002-4212-2618
}

\begin{abstract}
This paper deals with the fatigue assessment of cast steel defects in terms of macroscopic shrinkage porosity. Within preliminary studies, a generalized Kitagawa diagram GKD was established by numerical analyses of V-notched specimens with varying opening angles. It was experimentally verified by the application of the notch stress intensity factor (NSIF) concept on fatigue tests under rotating bending and axial loading. This paper continuous the work by an application of the GKD to real cast steel pores. At first, casting simulations are performed to design representative cast specimen geometries. The study focusses on macroscopic shrinkage pores with different spatial shapes. At second, fatigue tests under axial loading are conducted. Subsequent fracture surface analysis by light optical and scanning electron microscopy provides fracture mechanical based geometry parameters. Finally, the results of the experiments related to the failure relevant defect sizes are assessed by the GKD. In order to define an equivalent defect size of the complexly shaped defects, numerical crack growth analyses are performed demonstrating crack coalescence path tendencies. Summing up, the application of the NSIF approach based on a GKD shows a sound accordance to the experimental results and thus provides an engineering-feasible fatigue assessment method of cast steel components with macroscopic imperfections.
\end{abstract}

KEYwORDS. Generalized Kitagawa diagram; NSIF-concept; cast steel imperfections; crack initiation; crack path coalescence, numerical crack growth simulation.

\section{OPEN ACCESS}

Citation: Schuscha, M., Leitner, M., Stoschka, M., Pusterhfer, S., Meneghetti, G., Numerical crack growth study on porosity afflicted cast steel specimens, Frattura ed Integrità Strutturale, 48 (2019) 58-69.

Received: 30.11 .2018

Accepted: 30.12 .2018

Published: 01.04.2019

Copyright: (C) 2019 This is an open access article under the terms of the CC-BY 4.0, which permits unrestricted use, distribution, and reproduction in any medium, provided the original author and source are credited. 


\section{INTRODUCTION}

$\mathrm{T}$

oday's rapidly increasing requirements of lightweight construction and economical design enforce the application of local fatigue approaches. Despite high manufacturing standards of casting process technologies, the occurrence of shrinkage-based imperfections as well as sand, slag, or non-metallic inclusions is unavoidable. These casting process dependent, metallurgical properties occur within each cast part and significantly affect the local fatigue strength.

The assessment of such defects is commonly taken into account by threshold-based approaches like Murakami's Varea concept [1], which exhibits a proven match with experimental data [2, 3]. However, this concept does not take the spatial shape of the defect into account, but utilizes a characteristic length based on the imperfection area perpendicular to the maximum principal stress. In addition, adjacent flaws may interact and should be combined in order to support a conservative fatigue assessment [4]. Murakami's approach is generally applicable to imperfections of sizes smaller than one-thousand microns [5-7]. However, in case of cast steel, the irregularly shaped, shrinkage-based imperfections might be within several hundred up to thousands microns because of the increased solidus temperature and the limited feeding effectivity . Hence, this study encourages the use of a generalized Kitagawa diagram (GKD) to evaluate the local fatigue strength of arbitrary shaped, shrinkage-based imperfections for cast steel G21Mn5.

Usually, cyclically loaded notched components are designed based on their local fatigue resistance. Therefore, classical fatigue approaches utilize the stress concentration factor $\mathrm{K}_{\mathrm{t}}$, when full notch sensitivity is given. Otherwise, at the occurrence of partial notch sensitivity, $K_{t}$ can be corrected by a local support factor [8], which leads to the notch fatigue factor $K_{\mathrm{f}}$. Considering a component's notch with a root radius $\rho$ towards zero, notch mechanics based approaches are not any longer applicable $[9,10]$. Therefore, linear elastic fracture mechanics was adopted to assess the local stress field in front of cracks. The behaviour of small defects at the threshold regime as opposed to long cracks at was presented by Kitagawa and Takahashi [11]. As cracks possess an opening angle of zero, the crack tip related stress field for open cracks needs to be generalized to support the fatigue assessment of sharp notches in a uniform way. Considering Eq. 1 the factor $\mathrm{q}$ represents the materials internal wedge angle as is illustrated in Fig. 1. By determining the non-trivial solution, the expression exhibits the Williams' eigenvalue $\lambda$, which is used to calculate the stress singularity $\gamma=1-\lambda$.

$$
\sin \left(\lambda_{1,2} q \pi\right) \pm \lambda_{1,2} \sin (q \pi)=0
$$

Moreover, fundamental studies by Williams [12] as well as Gross and Mendelson [13] exhibited a link between the stress field in front of a notch tip, in terms of the plane problem, and its notch opening angle. The resulting parameters $\mathrm{K}_{\mathrm{I}} \mathrm{V}$ and $\mathrm{K}_{\mathrm{II}} \mathrm{V}$, defined as the notch stress intensity factors (NSIF) for mode one and mode two respectively, thus provide equivalent stress intensity factors for notches. For a component with a sharp V-notch, implying notch radius $\rho$ close to zero, under pure mode one loading $\sigma_{\theta \theta}$ (Fig. 1), the corresponding NSIF value can be calculated by Gross' and Mendelson's expression as:

$$
K_{I}^{V}=\sqrt{2 \pi} \lim _{r \rightarrow 0}\left[\sigma_{\theta \theta}(r, \theta=0) r^{1-\lambda_{t}}\right]
$$

Furthermore, studies extended the Kitagawa diagram to cover sharp and rounded V-notches [14-16]. Therefore, this approach can be uniquely applied to assess different types of notches, like blunt and sharp crack-like notches as well as notches with an opening angle greater than zero. Recent studies $[17,18]$ presented the utilization of energy-based approaches to assess the notch fatigue strength independently from their opening angle and mesh density [19, 20]. Thereby, the elastic stress-strain field in a fraction of the cross-section at the notch tip is taken into account for the determination of the local strain energy density. Depending on the notch opening angle, the evaluation area is defined as a circle or circular segment shape. Regarding cyclic loading, the size of this control area is coupled to the threshold stress intensity factor range $\Delta K_{I}^{V}$ and the fatigue limit range $\Delta \sigma_{0}$ of the base material and can be calculated as following [18]:

$$
\mathrm{R}_{c}=\left(\frac{\sqrt{2 e_{1}} \Delta K_{I}^{V}}{\Delta \sigma_{0}}\right)^{\frac{1}{1-\lambda_{1}}}
$$




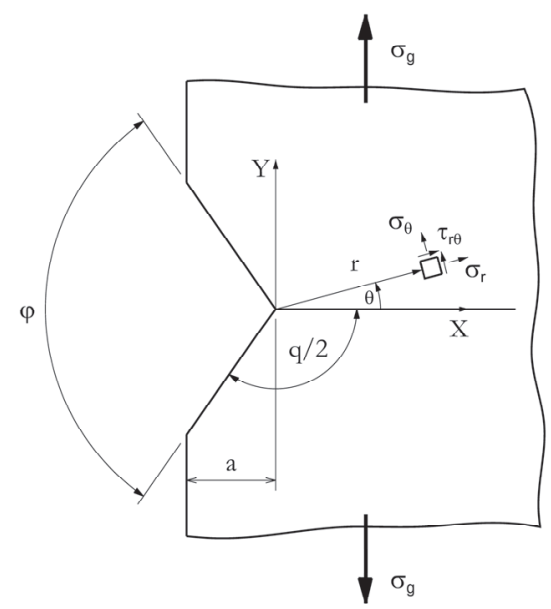

Figure 1: Structure weakened by a V-notch with opening angle $\varphi$ and notch depth $a$ subjected to mode I loading

Dealing with flaws, such as notches and especially macroscopic shrinkage porosities, where crack initiation and propagation may occur, an assessment of the materials short and long crack behaviour is indispensable. In general, the fatigue crack propagation threshold $\Delta K_{t h}$ can be understood as the condition at which a crack starts to grow or arrests assuming full-established crack closure effects. An important hypothesis regarding the long crack threshold is the partitioning into an intrinsic crack growth threshold value and an extrinsic fraction, which includes all mechanisms that are contributed from the crack flanks [21]. Consider a short crack that has not built-up any closure effects. In this state, only the materials crack growth resistance as effective threshold $\Delta K_{t b \text {, eff }}$ affects the propagation behaviour. Along with the crack growth several effects, such as plasticity-, roughness- and oxide-induced effects build up and raise the local propagation resistance until the intrinsic threshold value attains the long crack growth threshold stress intensity factor. This increase of the crack growth resistance over the crack length can be illustrated in the R-curve [22, 23] as it is shown in Fig. 2.

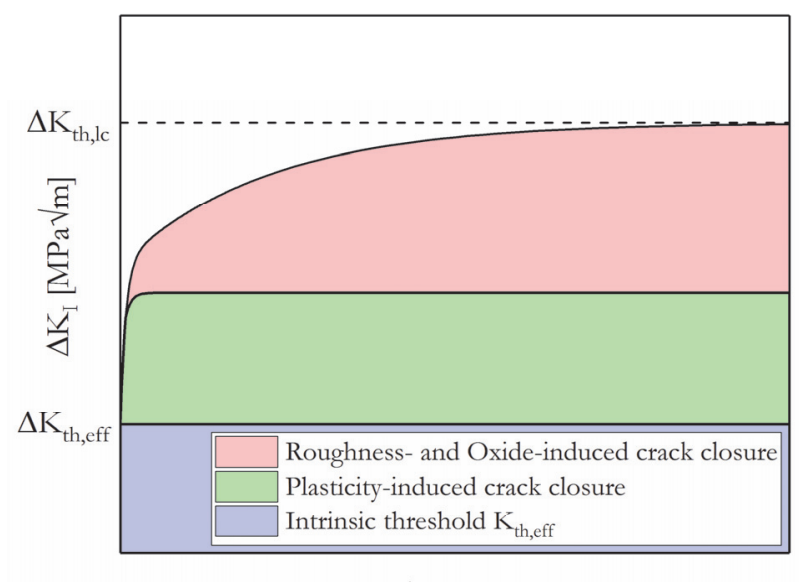

$\Delta \mathrm{a}[\mathrm{mm}]$

Figure 2: Representative structure of an R-curve

This paper deals with the basic applicability of the generalized linear elastic notch fracture mechanics towards casting defects of macroscopic porosity afflicted round specimens. The local fatigue assessment of such shrinkage-based imperfections is done by utilizing the GKD for G21Mn5 (1.1138) cast steel in normalized condition as follows.

- At first, threshold based material properties are obtained by single-edge-notch-bending and fatigue tests of the base material exhibiting no major casting defects. These data act as input parameters for the generalized Kitagawa- Takahashi diagram. The set-up of the numerical calculation is validated by fatigue tests covering several $\mathrm{V}$-notched S/N-curves with varying opening angles. Details are provided in preliminary studies, see [24, 25]. 
- At second, cast process simulation enables the design of an imperfection-afflicted specimen with characteristic defect geometry. Fatigue tests utilizing these designed specimens as well as extensive fracture surface analyses to characterize each defect characteristics are performed.

- At third, the characteristic spatial imperfection shape is measured by X-ray and computed tomography. The numerical crack growth is studied by utilizing the software tool Franc3D C for arbitrary shaped imperfections. In addition, the relation between the imperfection's area and the size of equivalent penny-shaped cracks is studied to determine a link between shrinkage-based porosities and a comparatively crack size.

- Finally, the fatigue test results of the cast specimens utilizing the equivalent defect sizes of the numerical crack growth analyses are validated with the preliminary designed GKD.

\section{DEVELOPMENT OF THE GENERALIZED KITAGAWA DIAGRAM}

$\mathrm{T}$ he generalized Kitagawa diagram (GKD) in the form presented by Atzori, Lazzarin and Meneghetti [16] requires two base material parameters, the plain fatigue limit $\Delta \sigma_{0}$ and the threshold stress intensity factor $\Delta K_{t h, l c}$. Therefore, experimental tests with un-notched specimens under rotating bending and axial loading at an alternating load ratio of $\mathrm{R}=-1$ were conducted [24]. In these cases, the cracks initiated from defect-free points from the specimens surface. The investigated base material is the cast steel G21Mn5 (grade ASTM 352) in normalized condition. Furthermore, crack growth tests were performed to establish the long crack stress intensity factor threshold value. These two parameters define the short crack region of the Kitagawa-Takahashi diagram, in which a crack of a size smaller than the materials intrinsic length $a_{0}$ is assumed to have no effect on the endurable high-cycle fatigue strength [26]. As the stress singularity of $\mathrm{V}$-notches depends on their notch opening angle, the threshold stress intensity factor for cracks can be transferred into an equivalent threshold notch stress intensity factor $\Delta K_{t b} V$. Thereby, three approaches, the finite-volume energy, fracture mechanical or point method, can be applied to determine the threshold value of the notch stress intensity factor NSIF, see [16]. After the link between $\Delta K_{t h, l c}$ and $\Delta K_{t b} V$ is established, the characteristic defect size $a_{0} V$ can be expressed as follows:

$$
a_{0}^{V}=\left(\frac{\Delta K_{t h}^{V}}{\sqrt{\pi} \Delta \sigma_{0}}\right)^{\frac{1}{\gamma}}
$$

Additionally, numerical analyses of $\mathrm{V}$-notched round specimens were performed under axial as well as bending load cases to determine the nominal NSIF values. To ensure the applicability of the presented formula, the specimens were modelled with a notch root radius equal to zero. Eq. 2 is utilized to calculate the corresponding NSIF value of each specimen type based on the local stress path beneath the notch tip. Furthermore, the determined notch stress intensity factor can be used to calculate an effective notch depth $a_{e f f}$, see (Eq. 5) [16], which equals the size of a notch in an infinite plate, similar to Griffith's crack model.

$$
K_{I}^{V}=\sigma_{g} \sqrt{\pi}\left(\alpha_{\gamma}^{1 / \gamma} a\right)^{\gamma} \quad a_{e f f}=\alpha_{\gamma}^{1 / \gamma_{a}}
$$

Based on the determined material and geometry parameters the GKD is set-up. Due to the calculated effective notch depth of each specimen geometry, an associated fatigue strength limit can be derived. Subsequently, experimental tests with the notched specimens were performed on a rotating bending machine as well as on an axial resonant test rig. The evaluated fatigue strength limits at a survival probability of fifty percent are related to their effective notch depth in the GKD. Based on the comparably small difference between the stress singularity of a crack and the V-notch with an opening angle $\varphi=45^{\circ}$, the NSIF of the $45^{\circ}$-notched specimen can be approximately treated like a crack of the same length. This leads to a change of only about one percent in the notch stress intensity factor value. As this reference value is taken as characteristic material length reference, a sound accordance between the estimated and experimental data with a deviation of only up to three percent is achieved even for differently notched samples under tension and bending [24]. 


\section{NUMERICAL CAST SIMULATION TO DESIGN REPRESENTATIVE SPECIMENS}

he idea to apply a GKD is to assess porosity afflicted cast steel components in a unique manner. Therefore, a representative specimen series needs to be designed facilitating cast simulation tools. Magma5C is used for the geometry modelling and casting process analysis.

Casting flaws are categorized into different types, like gas porosities, non-metallic inclusions and hot tears, whereby each type possesses different spatial extents and statistical distributions. The formation of shrinkage porosities is influenced by the inferior local feeding condition. This effect is utilized to study the probability of occurrence for macroscopic shrinkage defects on demand. Initially, the specimen geometry is defined with a test section diameter of $30 \mathrm{~mm}$ and a total length of $300 \mathrm{~mm}$. Based on this specification different models for the casting geometry are obtained.
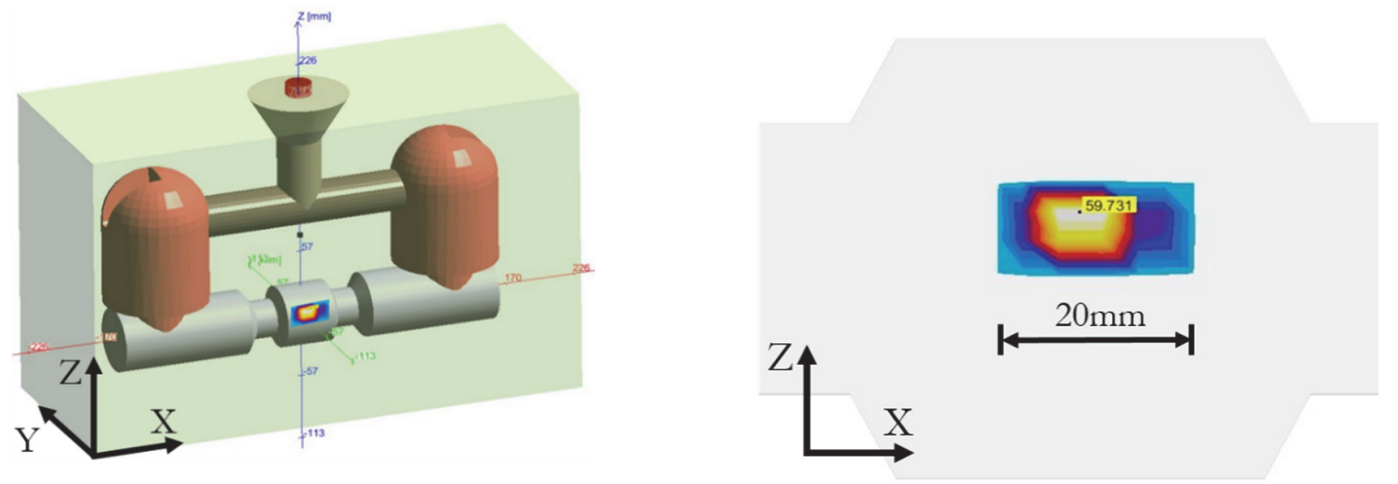

Figure 3: Representative moulding geometry (a) and resulting porosity within the testing section of the specimen (b)

The intended location of the purposeful generated macroscopic shrinkage porosity is in the centre of the specimen. Therefore, the geometry in the centre region is constricted on both sides by narrow cross sections to reduce the feeding capability of the centre area. Due to the delayed solidification in the centre volume, shrinkage porosity should occur intentionally. In order to develop different shapes of defects, a variation study of about four-hundred different geometry combinations is conducted. Thereby, the diameters and the lengths of the centre sections are varied to study the local feeding capability as well as the solidification time of the melt. Fig. 3(a) illustrates the mould geometry including the ingot, the gates, the feeders and the representative specimen, whereas Fig. 3(b) depicts the result of the simulation in terms of the total porosity in the centred sample region. The proposed defect exhibits dimensions of twenty by ten millimetre regarding length and diameter, respectively. Further, a vertical shift of around five millimetre can be expected, based on the static pressure during the solidification.
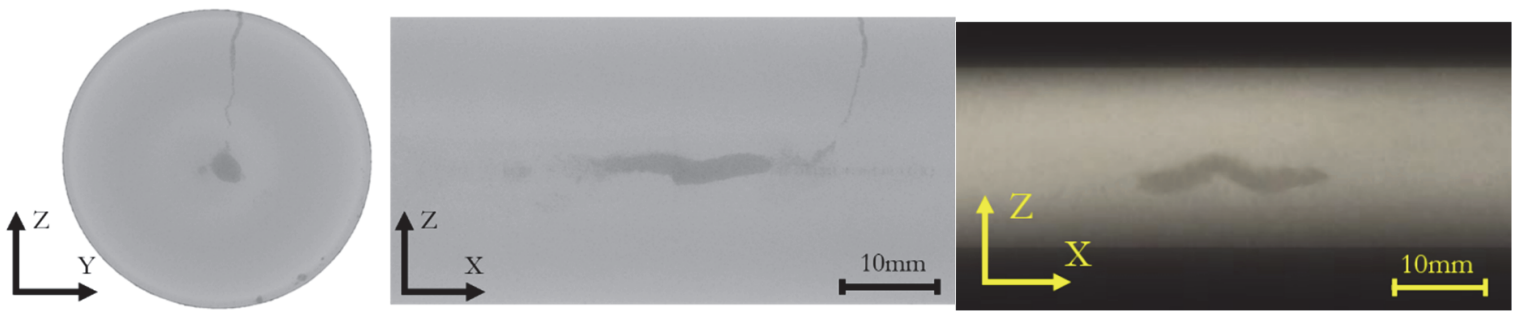

Figure 4: Computed tomography scan (a) and X-ray examination (b) of a porosity-afflicted specimen .

Subsequently to the cast simulation, the designed specimen are cast and heat-treated to justify a normalized microstructure in accordance to the aforementioned V-notched specimens. In order to assess the spatial porosity, X-ray shots are performed on each specimen. Characteristic specimens are investigated in depth by additional computed tomography.

The X-ray inspection reveals cast defects located in the centre of the specimens as desired. The shrinkage dimensions range in axial length from ten to thirty millimetres, a cylindrical diameter up to four millimetres and, in some cases, a shift of several millimetres parallel to the axis is obtained. Further, the results partially show additional single defects located at the narrow cross-sections, which feature an arbitrary shape. A comparison of the non-destructive-testing methods X-ray and computed tomography highlight a sound agreement of the centre porosity results regarding defect dimensions and 
spatial location. Fig. 4 illustrates the computed tomography as well as the X-ray examination of a defect found in the specimen's centre. This defect exhibits a length of twenty-nine and a diameter of four millimetres.

\section{EXPERIMENTAL INVESTIGATIONS}

$\mathrm{I}$

$\mathrm{n}$ order to assess the cast material defects, fatigue tests on an axial fatigue test rig under a tumescent stress ratio of $\mathrm{R}=0$ are performed. The test abort criterion is total burst failure in the finite-life region. Moreover, at ten million load cycles the specimen is defined as run-out and subsequently re-inserted in the finite life region.

Despite the comparably huge size of the pores located in the centre of specimens, which were properly detected by nondestructive testing, first tests exhibited crack initiation by an arbitrary shaped crack-like defect located at the former narrow cross section. This crack-like defect type samples are labelled as failure type FT\#A. Subsequently, experimental investigations of specimens, which exhibit no such additional crack-like defects, show an increased fatigue resistance determined by their pore-like imperfection within the centre of the specimen. These samples, featuring an elongated macroscopic pore shape, are further on denoted as FT\#B. Fig. 5(a) and 5(b) illustrate representative fracture surfaces for a FT\#A and FT\#B, respectively.
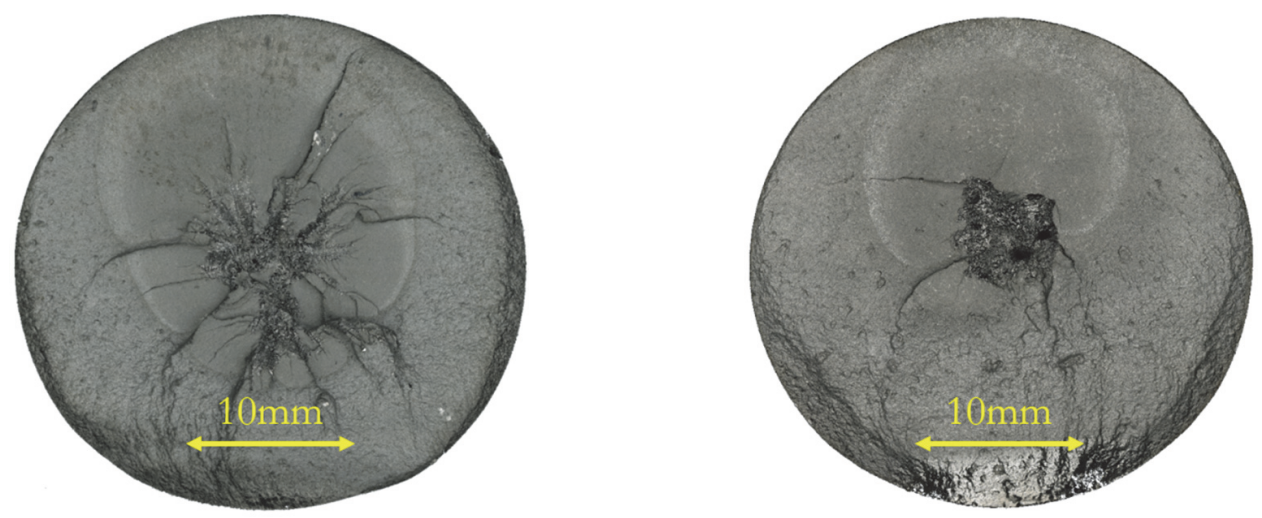

Figure 5: Fracture surface of a FT\#A defect (specimen "GP1") (a) and of a FT\#B defect (specimen “GP21”) (b)

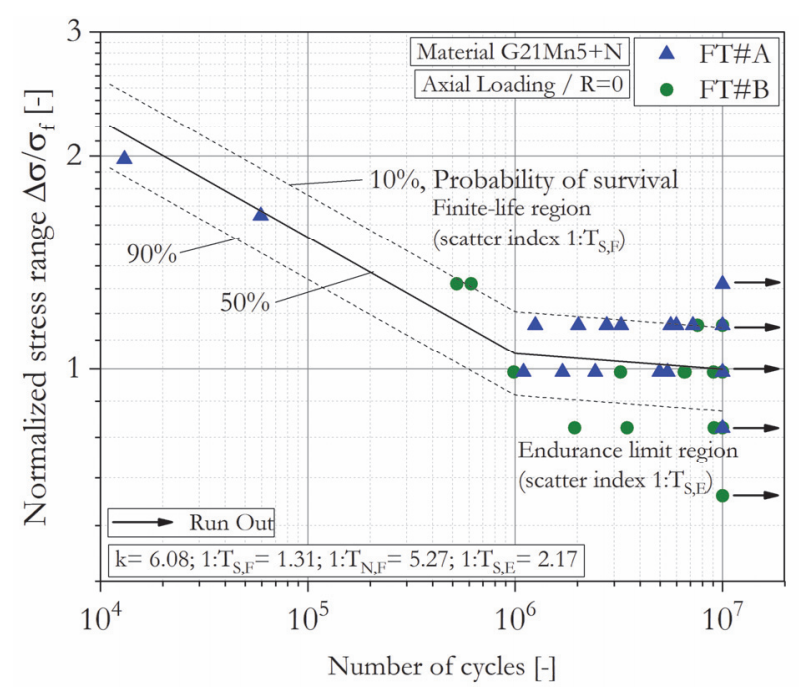

Figure 6: S-N curve of the large-scale specimen tests

Fig. 6 illustrates the normalized results of the experimental tests, related to their long-life fatigue strength limit. Based on the defect shape, spatial orientation and axial position within the specimen, the investigated samples are split into two failure types, as explained before. Concerning a NSIF-based defect assessment of the large-scale samples, it is necessary to assign the defect types to their equivalent opening angle within the GKD. Within the subsequent section, the numerical 
crack growth analysis is performed in order to define an equivalent defect size for each failure type. Finally, the fatigue test data utilizing the representative defect size values are included in the GKD and an equivalent opening angle is assigned. This procedure enables a link between the small-scale V-notched specimen test results, which were used to set-up the GKD, and the defect-afflicted cast specimens within this study.

\section{NUMERICAL CRACK GROWTH ANALYSIS}

1

he numerical crack propagation analysis utilizing a three-dimensional model is illustrated for three samples of the defect type FT\#A and for one sample of FT\#B. This strategy is reasonable, as FT \#A specimen possess quite irregularly shaped casting imperfections and enforces simulation of multiple geometries. As FT\#B exhibits a more rational geometric shape it can be already judged by a single simulated crack growth study. Aiming at an almost realistic defect shape, the analysed flaw geometries are taken from the fracture surfaces images. Despite the overall threedimensional shape of the casting defect, the planar geometry is obtained by light optical microscopy supported fracture surface analysis and subsequently inserted as a planar crack into a three-dimensional model of the representative specimen. In order to obtain the geometry of the defect, an image post-processing tool is utilized to mark the defect boundaries on the fracture surface images. Subsequently, these marks are used to maintain the boundary points around the defects perimeter. Initial numerical crack growth calculations resulted in sometimes-distorted elements due to partially small radii along the perimeter. As these lead to a negative Jacobian matrix, the local defect curvature is evaluated in every point at the perimeter and adjusted to establish a solvable crack growth model. Fig. 7 depicts the defect geometry on the fracture surface of a representative specimen and further, the adjusted simplified shape that is utilized in the numerical simulation. It should be noted that such a complex, star-shaped defect structures within the large-scale cast specimen demands a precise crack growth simulation run considering shape, mesh size and local curvature radii. Therefore, an equivalent elementary geometry, like a penny-shaped crack, is utilized as an engineering-feasible substitution to study the propagation behaviour.

At first, a crack growth simulation based on the adjusted defect geometry is performed until a circular shape is obtained by incremental crack growth. The aim of these investigations is to derive a penny-shaped crack, which exhibits an 'identical' lifetime until burst failure. Consequently, the projected area of the adjusted defect geometry is utilized to determine an equivalent circle diameter, abbreviated as ECD. Subsequent, various penny-shaped models are set-up with different ratios of the ECD as the diameter of the initial crack size, respectively. Fig. 7 additionally illustrates a penny shaped crack for the given defect shape with an area ratio equal to one. The crack growth simulation is performed under a tumescent load ratio in accordance to the fatigue tests. Further on, the simulation utilizes the experimentally determined fracture mechanical parameters in terms of the modified NASGRO equation [23].
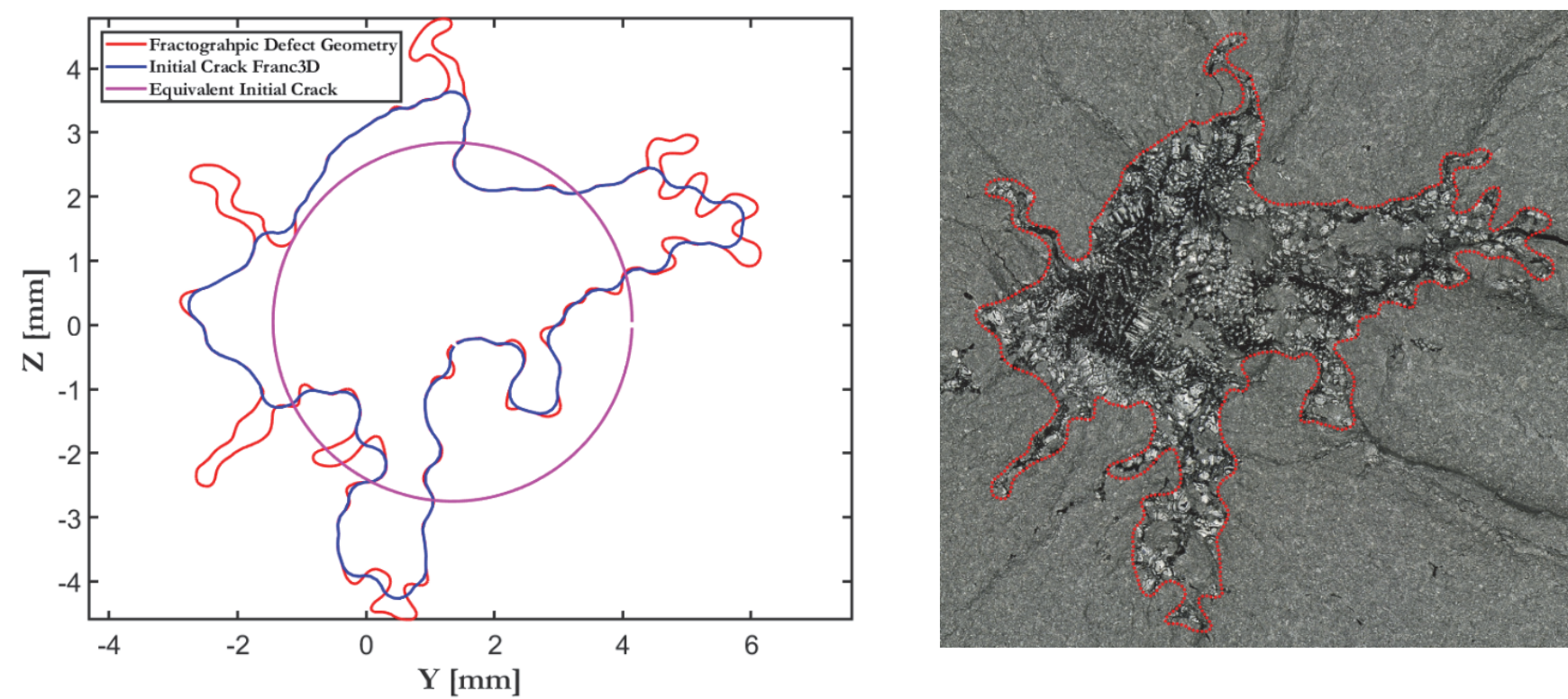

Figure 7: Fractographically-based versus adjusted defect geometry and equivalent penny-shaped crack size (a) and fracture surface of the related sample (b) 
The results of each incremental simulation step are utilized to calculate the local stress intensity factors and the crack growth rate at every point at the current crack boundary. Subsequently, the crack shape is locally adjusted based on the assessed fracture mechanical values and re-inserted into the numerical model of the large-scale specimen. This procedure repeats within a loop until the shape of the crack front of the arbitrary defect is similar to the shape and size of the apparent crack size on the fracture surface before burst failure. As a comparison, the penny-shaped equivalent cracks also are assessed by the numerical simulation under the same load conditions to evaluate an equivalent circle diameter (ECD) crack size, which exhibits the same lifetime as the experiment of the defect-afflicted specimen.

Consequently, this simulation strategy is performed for the four different defect geometries reflecting failure type FT\#A and FT\#B. Fig. 8(a) depicts the normalized total lifetime of the experimental investigations and the results of the numerical analysis. One can see that in the case of the arbitrary star-shaped defect, "GP1" as a member of FT\#A, a huge difference between the experimental and simulation result exists. In detail, the result indicates that only about $6 \%$ of the total lifetime is related to the crack growth and $94 \%$ to the crack initiation. This ratio leads to the statement, that the numerical analysis, which only takes the crack growth lifetime into account, is far more conservative than the real fatigue behaviour of the shrinkage porosity. As the GKD is a threshold-based fatigue assessment method utilizing a certain initial crack size, the used initial ECD needs to be adjusted targeting an equal lifetime of the penny-shaped defect and the experimental results. This empiric approach leads to a sound agreement of the lifetime values if a factor of one quarter of the ECD is used as initial crack size in case of failure type FT\#A defects.

Concerning FT\#B, which is considered by the defect of the sample "GP21", the initial analysis of ECD exhibits a larger ratio of nearly $15 \%$ of the experimental lifetime due to a higher average crack growth rate. As it is illustrated in Fig. 8 (b) the ECD/2, which represents an initial crack size of one-half of the equivalent circle diameter of the fractographic defect, attains almost $80 \%$ of the total lifetime. A further simulation using $3 / 8$ of the ECD as initial crack size, which is not illustrated in Fig. 8(b), overshoots the experimental lifetime by nearly $60 \%$ in non-conservative way. Therefore, the correction factor for FT\#B defects regarding the assessment by the GKD with a conservative distinction is defined as one-half of the equivalent circle diameter as this value revealed a satisfying and still conservative match to the experimentally evaluated lifetime.
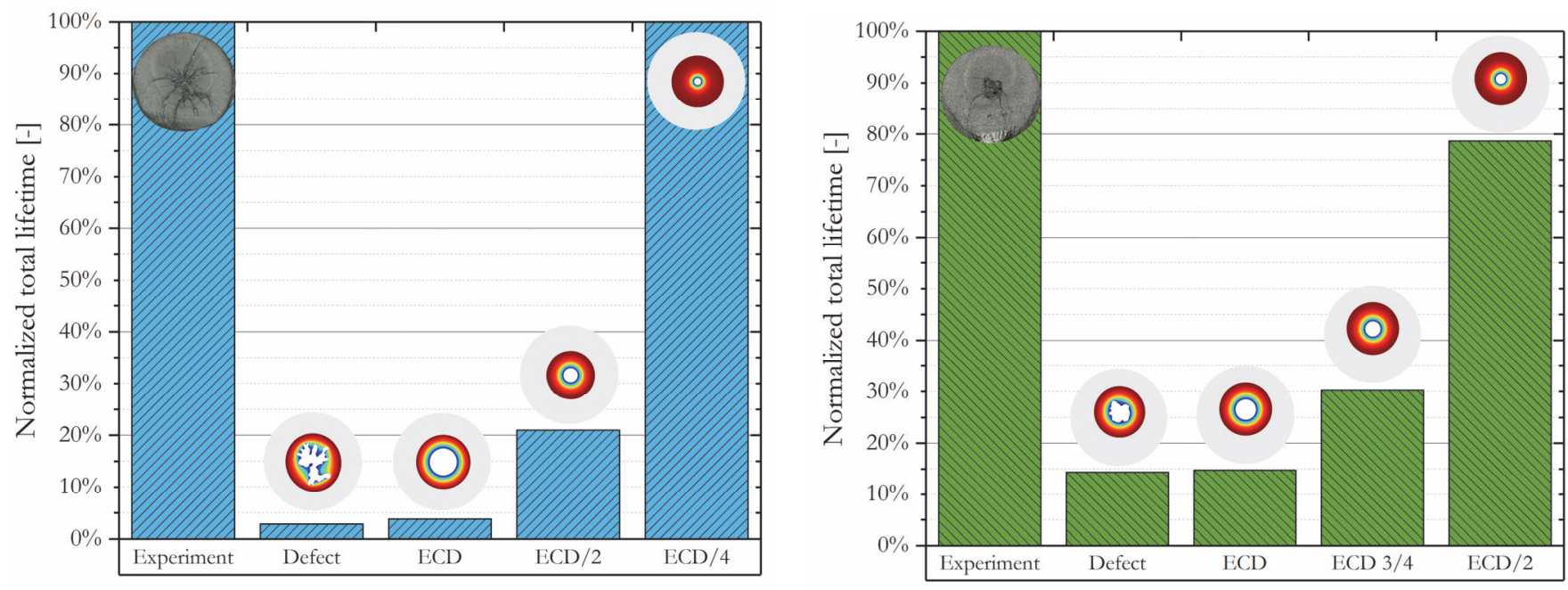

Figure 8: Simulation results of the FT\#A model "GP1" (a) and the FT\#B model "GP21" (b)

Figs. 9(a) and (b) illustrate the life cycle fractions for "GP1" and "GP21", respectively. The coloured circular rings represent the local life cycle fraction related to the total lifetime. Based on the numerical results, the crack propagation can be subdivided into a subsection until the crack attains a circular shape and into another subsection, where a steady circular crack growth occurs. In terms of the arbitrary shaped "GP1", a convex hull of the defect is attained at approximately $80 \%$ of the total life cycles. The residual part of the total lifetime of $20 \%$ compromises a steady penny-shaped crack growth. Considering the steady-shaped defect, Fig. 8(b), almost $25 \%$ of the lifetime is needed to achieve the circular shaped hull and $75 \%$ can be assigned to the entirely circular crack propagation.

In general, the first cycles of the simulation show an increased growth rate as well as stress intensity factors at the interior curved sections of the crack. Due to the rising crack length and the consideration of the modified NASGRO equation [23], crack closure effects build up, which locally reduce the crack growth rate. 

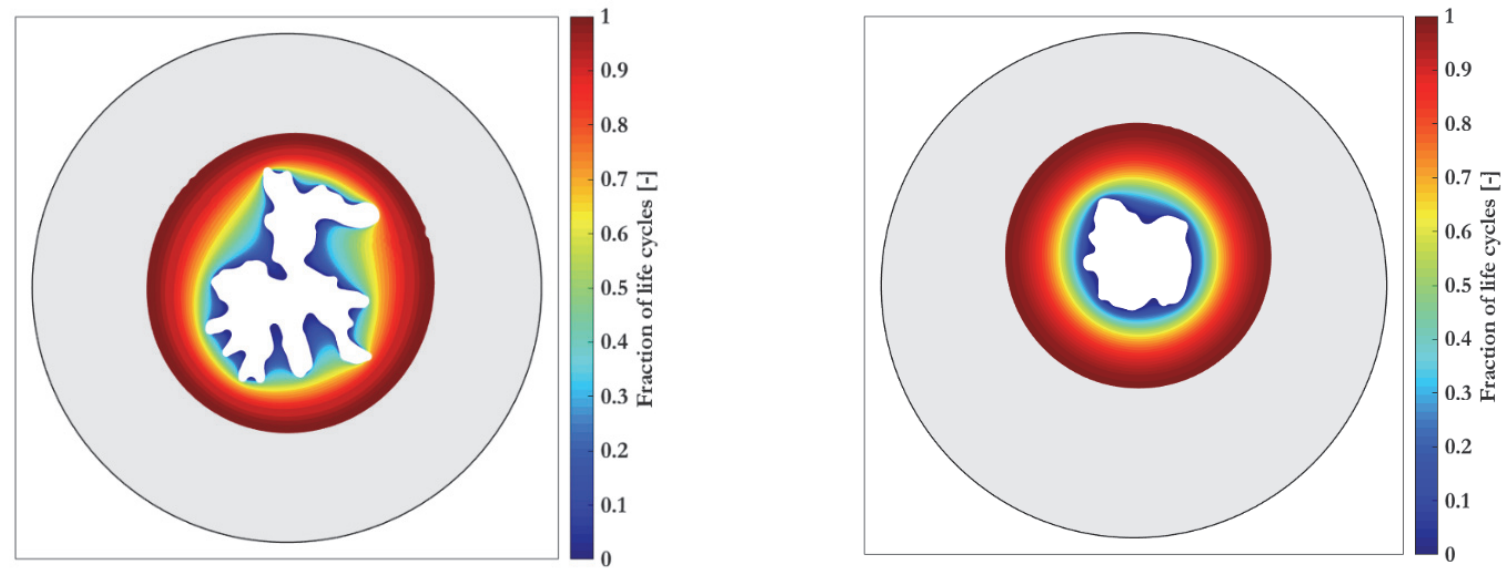

Figure 9: Life cycle fractions of the defect simulations of the FT\#A model "GP1” (a) and the FT\#B model "GP21" (b)

Fig. 10(a) and (b) illustrate the change in crack growth rate during the crack propagation analysis. The colour bar runs from yellow, which correlates with a small propagation rate, to dark red, which corresponds to a high crack growth rate. As one can see, the growth rate drops after an initial high crack propagation stage, but still increases the crack size. Fig. 10(b) depicts the maximum stress intensity factor and the corresponding crack growth rate based on the modified NASGRO-equation. Aiming at a more intuitive explanation, Fig. 10(a) is extended by three points, marked with "1", "2" and "3", that represent the location of the maximum crack growth rate at each simulation step. At the beginning of the simulation, the finite element analysis resulted in a maximum stress intensity factor range of $\Delta K \sim 17 M P a \sqrt{ }$, which is located in the sharp inner region of the star-shaped pore and labelled as point "1". In this first simulation step, no crack propagation occurred so far. Thus, the calculation of the corresponding $d a / d N$ values are utilized based on the limiting curve valid for a short crack. Due to crack propagation in the subsequent analysis steps, the local curvature of the crack front is smoothened accordingly to the local $\Delta K_{I}$ values around the perimeter. Although the local crack length is increasing from point "1" to point " 2 ", which obviously would lead to a raise of the stress intensity factor range, the smoothening of the curvature exceeds the influence of crack propagation; hence, in total the local stress intensity factor range decreases. Further on, the crack growth leads to a build-up of crack closure effects, which decrease the crack propagation rate. Due to these two fracture mechanical effects, the maximum stress intensity factor range drops to a value of $\Delta K \sim 13 \mathrm{MPa}{ }_{\mathrm{m}}$ and the crack propagation rate decreases by a factor of five, which is outlined by the path from point "1" to "2". At point "2", the local crack curvature is smoothened and no further decrease of the local $\Delta K$ is observed. Moreover, the elliptical crack continuously grows towards a fully circular shape against the long-crack limiting curve, which is illustrated by the path from point "2" to " 3 ". Finally, the long-crack limiting curve including the fully built-up crack closure effects is reached at point " 3 ", which is followed by the Paris regime.
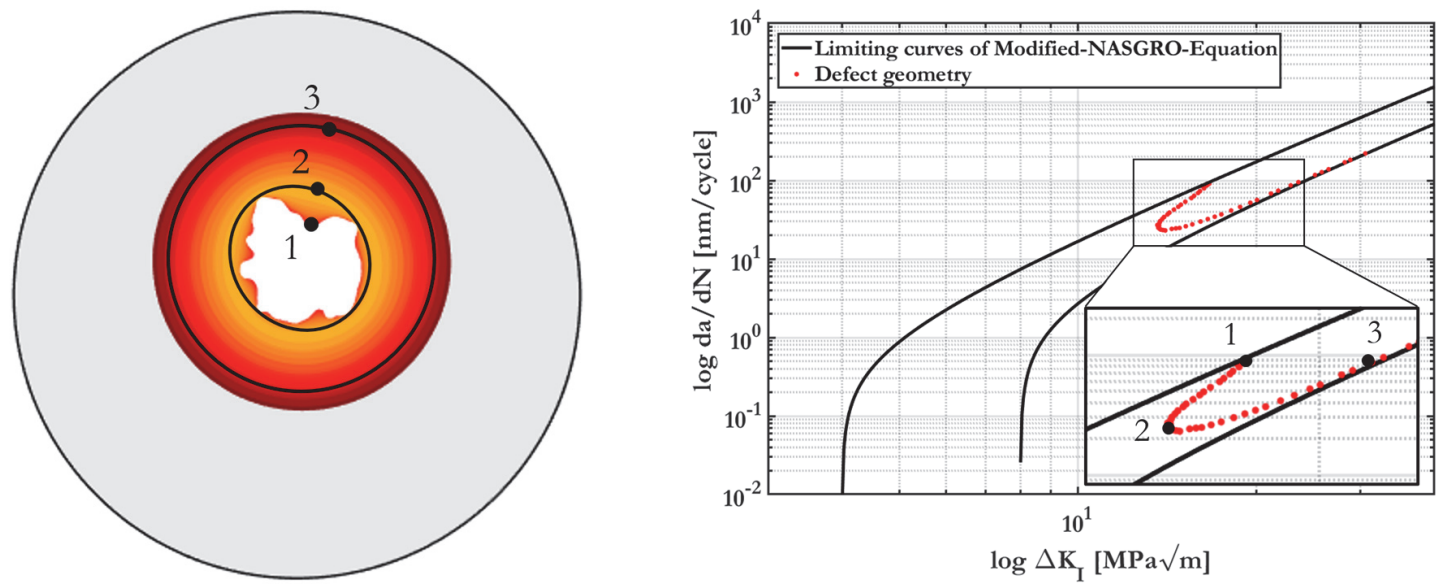

Figure 10: Illustration of the general defects growth behaviour during the analysis (a) and development of the maximum growth rates along the evaluation path " $1-3$ " (b) 
Furthermore, the crack-growth results link the fractographic defect shape and the penny-shape geometry in terms of the life cycles. Based on the outcome of this numerical study, the replacement of an arbitrary shrinkage defect by a pennyshaped crack that exhibits either one-quarter for FT\#A, or seven-eighths for FT\#B, as engineering feasible approximation, is recommended.

\section{FATIGUE ASSESSMENT OF POROSITY-AFFLICTED CAST STEEL SPECIMENS USING THE GKD}

$\mathrm{B}$

ased on the results of the numerical crack growth analysis, the effective sizes are determined by the corrected equivalent circle diameters for FT\#A and FT\#B, respectively. As illustrated in Fig. 11(a), the results feature a sound agreement with the GKD.
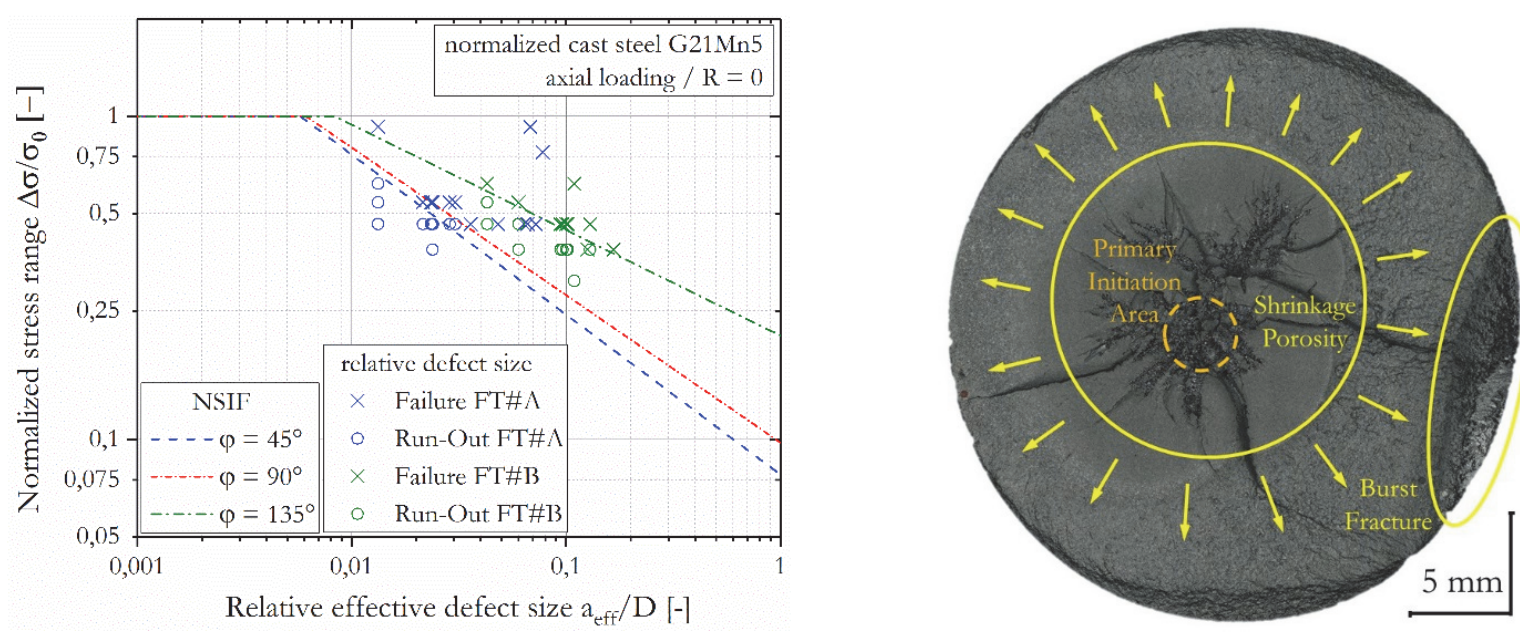

Figure 11: Normalized GKD of experimental tests (a) and LOM analysis of fracture surface with FT\#A defect (b)

The fracture surfaces of the tested specimens are analysed by light optical microscopy (LOM) and by scanning electron microscopy (SEM) in order to evaluate the size of the defects as well as to determine the crack initiation. As illustrated in Fig. 11(b), the failure of the specimens initiates on multiple, partially connected shrinkage porosity locations and coalescence by further crack growth. To derive a proper technical crack initiation area, it is necessary to propose primary crack initiation areas that lead to conservatively matching mean fatigue life, which is achieved by the previously investigated numerical analysis. It should be noted that analytical solutions exist for standardized imperfections such as elliptical crack shapes, but as the studied imperfections exhibit multiple crack initiations of arbitrary shapes, a numerical crack propagation study is sometimes unavoidable to obtain a comparative, effective penny-shaped defect.

\section{SUMMARY AND CONCLUSIONS}

$\mathrm{F}$ irstly, a generalized Kitagawa-Takahashi (GKD) diagram is set-up for cast steel G21Mn5. It is based on experimental fatigue tests under rotating bending and axial loading of $\mathrm{V}$-notched specimens. The corresponding notch-stress-intensity-factors unify varying notch opening angles and imperfection sizes into a threshold-based fatigue assessment.

Secondly, numerical casting simulations for the development of representative specimens are conducted. Thereby, different model geometries are simulated to obtain different defect shapes and sizes. In order to enforce the occurrence of shrinkage porosity, the centre domain of the specimen is constricted by narrow cross section on both sides.

Thirdly, representative cast specimens are examined by X-ray, ultrasonic testing and computed tomography. Fatigue tests under axial loading reveal two different failure types based on a crack-like imperfection or a more globular porosity.

Finally, fracture mechanical simulations are conducted to study the crack growth behaviour of arbitrary, see FT\#A, and more regularly shaped defects, see FT\#B. Moreover, an equivalent penny-shaped crack size that exhibits the same fracture mechanical lifetime, as the related defect is deducible by introducing failure-type based corrections factors for the 
equivalent defect diameter. Therefore, the geometry of the defects are obtained from fractographic analysis as reference. Based on the fracture mechanical results, a factor of one-quarter is evaluated for the calculation of the effective defect size regarding the GKD for FT\#A defect types and a factor of one-half for more steadily-shaped defects representing FT\#B. Based on these empirically determined correction factors, the investigated cast steel samples of these two characteristic failure types are assessed by the aid of the GKD.

Ongoing work focusses on the utilization of local strain or energy-based approaches to take the crack initiation lifetime more properly into account. The importance of crack initiation based fatigue assessment by local approaches is also visible in the presented fraction of total lifetime. Star-shaped FT\#A-samples possess a distinctively increased defect perimeter compared to the more circular FT\#B-specimen and thus the amount of crack initiating lifetime is more pronounced. Summing up, the presented approach features a comprehensive methodology to assess fatigue life of macroscopic cast steel defects, which will be investigated further especially in regard of classification and effect study of their complexly shape in terms of spatially varying load vector and projected defect area.

\section{ACKNOWLEDGEMENT}

7 he financial support by the Austrian Federal Ministry for Digital and Economic Affairs and the National Foundation for Research, Technology and Development is gratefully acknowledged.

\section{REFERENCES}

[1] Murakami, Y. (2002). Metal Fatigue: Effects of Small Defects and Nonmetallic Inclusions, Elsevier.

[2] Beretta, S., Blarasin, A., Endo, M., Giunti, T., Murakami, Y. (1997). Defect tolerant design of automotive components, Int. J. Fatigue, 19, pp. 319-333. DOI: 10.1016/S0142-1123(96)00079-5.

[3] Murakami, Y., Yamashita, Y. (2014). Prediction of Life and Scatter of Fatigue Failure originated at nonmetallic Inclusions, XVII International Colloquium on Metallic Fatigue of Metals (ICMFM17), pp. 6-11. DOI: 10.1016/j.proeng.2014.06.214.

[4] Åman, M., Okazaki, S., Matsunaga, H., Marquis, G.B., Remes, H. (2017). Interaction effect of adjacent small defects on the fatigue limit of a medium carbon steel, Fatigue Fract. Eng. Mater. Struct., 40, pp. 130-144. DOI: $10.1111 /$ ffe.12482.

[5] Murakami, Y. (2012). Material defects as the basis of fatigue design, Int. J. Fatigue, 41, pp. 2-10. DOI: 10.1016/j.ijfatigue.2011.12.001.

[6] Krewerth, D., Lippmann, T., Weidner, A., Biermann, H. (2016). Influence of non-metallic inclusions on fatigue life in the very high cycle fatigue regime, Int. J. Fatigue, 84, pp. 40-52. DOI: 10.1016/j.ijfatigue.2015.11.001.

[7] Yamashita, Y., Murakami, Y. (2016). Small crack growth model from low to very high cycle fatigue regime for internal fatigue failure of high strength steel, Int. J. Fatigue, 93, pp. 406-414. DOI: 10.1016/j.ijfatigue.2016.04.016.

[8] Peterson, R.E. 1959). Notch sensitivity, (In: Sines G, Waisman JL, editors. Metal fatigue. New York: MacGraw-Hill, pp. 293-306.

[9] Frost, N.E., Marsh, K.J., Pook, L.P. (1974). Metal Fatigue, Oxford University Press, Oxford.

[10] Smith, R.A., Miller, K.J. (1978). Prediction of fatigue regimes in notched components, Int. J. Mech. Sci., 40, pp. 201 206. DOI: $10.1016 / 0020-7403(78) 90082-6$.

[11] Kitagawa, H., Takahashi, S. (1976). Applicability of fracture mechanics to very small cracks in the early stage, Proceedings of the $2^{\text {nd }}$ International Conference on Mechanical Behaviour of Materials, pp. 627-631.

[12] Williams, M.L. (1952). Stress Singularities Resulting From Various Boundary Conditions in Angular Corners of Plates in Extension, J. Appl. Mech., 19, pp. 526-528.

[13] Gross, B., Mendelson, A. (1972). Plane elastostatic analysis of V-notched plates, Int. J. Fract., 8, pp. 267-276. DOI: $10.1007 / \mathrm{BF} 00186126$.

[14] Atzori, B., Lazzarin, P. (2001). Notch Sensitivity and Defect Sensitivity under Fatigue Loading: Two Sides of the Same Medal, Int. J. Frac., 107, pp. 1-8. DOI: 10.1023/A:1007686727207.

[15] Atzori, B., Lazzarin, P., Meneghetti, G. (2003). Fracture mechanics and notch sensitivity, Fatigue Fract. Eng. Mater. Struct., 26, pp. 257-267. DOI: 10.1046/j.1460-2695.2003.00633.x. 
[16] Atzori, B., Lazzarin, P., Meneghetti, G. (2005). A unified treatment of the mode I fatigue limit of components containing notches or defects, Int. J. Fract., 133, pp. 61-87. DOI: 10.1007/s10704-005-2183-0.

[17] Glinka, G. (1985). Energy density approach to calculation of inelastic strain-stress near notches and cracks, Eng. Fract. Mech., 22, pp. 485-508. DOI: 10.1016/0013-7944(85)90148-1.

[18] Lazzarin, P., Zambardi, R. (2001). A finite-volume-energy based approach to predict the static and fatigue behavior of components with sharp V-shaped notches, Int. J. Fracture, 112, pp. 275-298. DOI: 10.1023/A:1013595930617.

[19] Campagnolo, A., Meneghetti, G., Berto, F. (2016). Rapid finite element evaluation of the averaged strain energy density of mixed-mode (I+II) crack tip fields including the T-stress contribution, Fatigue Fract. Eng. Mater. Struct., 39, pp. 982-998. DOI: $10.1111 /$ ffe.12439.

[20] Meneghetti, G., Campagnolo, A., Berto, F. (2016). Averaged strain energy density estimated rapidly from the singular peak stresses by FEM: Cracked bars under mixed-mode (I+III) loading, Eng. Fract. Mech., 167, pp. $20-33$. DOI: 10.1016/j.engfracmech.2016.03.040.

[21] Ritchie, R.O. (1988). Mechanisms of fatigue crack propagation in metals, ceramics and composites, Mater. Sci. Eng., A, 103(1), pp. 15-28. DOI: 10.1016/0025-5416(88)90547-2.

[22] Tanaka, K., Akiniwa, Y. (1988). Resistance-curve method for predicting propagation threshold of short fatigue cracks at notches, Eng. Fract. Mech., 30(6), pp. 863-876. DOI: 10.1016/0013-7944(88)90146-4.

[23] Maierhofer, J., Pippan, R., Gänser, H.-P. (2014). Modified NASGRO equation for physically short cracks, Int. J. Fatigue, 59, pp. 200-207. DOI: 10.1016/j.ijfatigue.2013.08.019.

[24] Schuscha, M., Leitner, M., Stoschka, M. (2018). Development of a generalized Kitagawa diagram for cast GS21Mn5 steels, Matec Web of Conferences 165, 14010. DOI: 10.1051/matecconf/201816514010.

[25] Schuscha, M., Leitner, M., Stoschka, M., Meneghetti, G. (2018). Application of the generalized Kitagawa diagram on cast steel, Proceedings of the $6^{\text {th }}$ International Conference on Crack Paths.

[26] El Haddad, M.H., Topper, T.H., Smith, K.N. (1979). Fatigue Crack Propagation of Short Cracks, Eng. Fract. Mech., 101(1), pp. 573-584. DOI: $10.1115 / 1.3443647$. 